\title{
Management of people with challenging behaviour
}

\section{Kiriakos Xeniditis, Ailsa Russell \& Declan Murphy}

The term 'challenging behaviour' was introduced in North America in the 1980s, and was originally used to describe problematic behaviours in people with 'mental retardation' (learning disabilities). Challenging behaviour can, however, occur across the intellectual spectrum, being particularly prevalent in populations with psychiatric disorder.

A widely accepted definition of challenging behaviour is:

"culturally abnormal behaviour of such an intensity, frequency or duration that the physical safety of the person or others is likely to be placed in serious jeopardy, or behaviour which is likely to seriously limit use of, or result in the person being denied access to, ordinary community facilities" (Emerson, 1995).

Previously used terms included 'behavioural disturbance', 'problem behaviour', 'maladaptive behaviour', 'aberrant behaviour' and 'behavioural abnormalities'.

The relationship between psychiatric diagnoses and challenging behaviour is a complex one and is represented graphically in Fig. 1. Challenging behaviour can occur in the absence of a psychiatric disorder and not all people with mental illness exhibit challenging behaviour. The overlapping area between 1 and 2 represents patients with challenging behaviour and psychiatric disorder. In some people, challenging behaviour may be an essential diagnostic criterion (e.g. eating disorders, personality disorders, paraphilias, etc.) or a secondary feature (e.g. self-injury in depression, aggression secondary to persecutory delusions in schizophrenia and wandering in dementia). Of course, challenging behaviour may simply coexist and often pre-date a psychiatric disorder (e.g. a violent offender who subsequently develops a depressive illness). Learning disabilities and autistic spectrum disorder may also be present in various combinations of comorbidity. The overlap between 1 and 4 represents those with dual diagnosis (mental illness and learning disabilities).

The part of 3 that lies outside 4 represents people with high functioning autism and Asperger's syndrome who may or may not have mental illness and/or challenging behaviour.

\section{Epidemiology}

The prevalence of challenging behaviour has been most extensively studied in learning disability populations, reflecting the longer established use of the term in this field. Prevalence studies of challenging behaviour in people with learning disability vary widely in their findings, reporting rates between 5.7\% (Qureshi \& Alborz, 1992) and 14\% (BorthwickDuffy, 1994). This probably reflects the different criteria used for case identification (for both learning disability and challenging behaviour) and the differences in the target populations (hospital and day centre communities). None the less, there is consensus that: males are more likely to be identified as having challenging behaviour than females; the overall prevalence increases with age during childhood, reaches a peak during the age range 15-34

Kiriakos Xeniditis is a consultant psychiatrist in the South London and Maudsley Trust; his research interests include the aetiology and management of challenging behaviour. Ailsa Russell is a lecturer in clinical psychology at the Institute of Psychiatry (Department of Psychological Medicine, Institute of Psychiatry, De Crespigny Park, Denmark Hill, London SE5 8AF) and an honorary clinical psychologist at the Maudsley Centre for Behavioural Disorders. Her research interests include the neuropsychological, diagnostic and demographic factors associated with challenging behaviour. Declan Murphy is Professor of Psychiatry and Brain Maturation in the Department of Psychological Medicine at the Institute of Psychiatry, an honorary consultant psychiatrist for the Royal Bethlem and Maudsley Hospitals and Clinical Director of the Maudsley Centre for Behavioural Disorders. His research interests include the biological determinants of developmental and degenerative brain disease. 


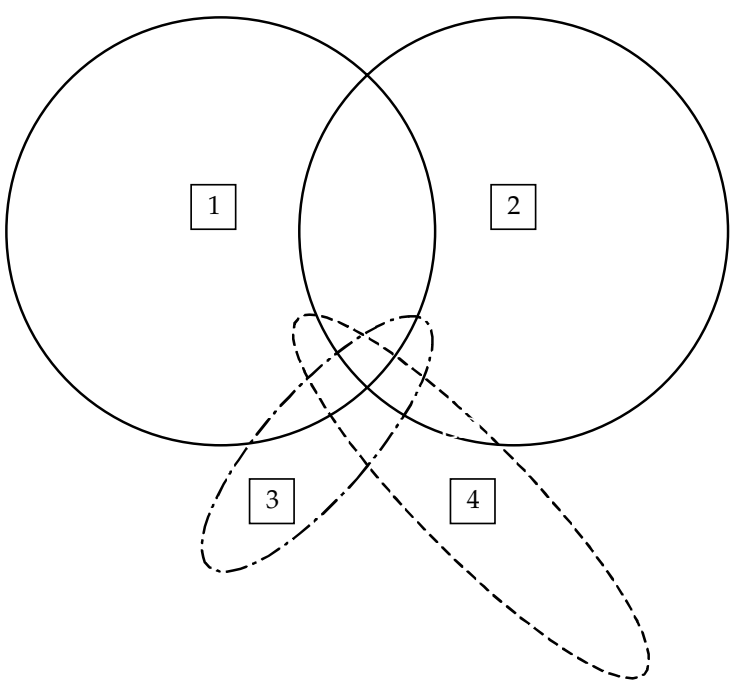

1, mental illness including personality disorder 2, challenging behaviours; 3 , autistic spectrum disorders; 4, learning disabilities

Fig. 1 The relationship between psychiatric diagnosis and challenging behaviour

years, and then declines; and the prevalence of challenging behaviours such as aggression and selfinjurious behaviour (SIB) is greater in people with more severe learning disability (for a review see Emerson, 1995).

In the general adult mental health literature, epidemiological studies of challenging behaviour as a single clinical entity are scarce, owing to the greater heterogeneity of clinical conditions subsumed under the title challenging behaviour (Box 1). There are, however, studies examining prevalence rates of specific problem behaviours in adult mental health settings. For example, Steinart et al (1999), in a study investigating the prevalence of aggressive behaviour in acute in-patient settings, found that $75 \%$ of the men in the sample and $53 \%$ of the women exhibited some type of aggressive behaviour (including harm to self) during their first or subsequent admission. A large number of studies have focused on the prevalence of substance misuse in clinical populations, commenting on its particular challenge for services. Regier et al (1993) established a prevalence of $29 \%$ for comorbid addictive disorders. More recent studies have established higher rates of substance misuse among patients with severe mental disorder, with $36.3 \%$ of patients having comorbid substance misuse, which is associated with greater use of in-patient services (Menezes et al, 1996). This is of importance because comorbid substance misuse is associated with an increased prevalence of violent behaviour and criminal offending in people with mental health problems.

\section{Aetiology}

Management of challenging behaviour should follow an aetiological rationale whenever possible. As challenging behaviour is not a unitary nosological entity, no single common cause for it can be identified. For example, the aetiology of self-injury or violence is most likely different from that of disorders of sexual behaviour. Moreover, for each individual behaviour, the causation is almost invariably multi-factorial. Thus, the causes of challenging behaviour are best studied using a bio-psychosocial model to examine the different influences on the development and maintenance of challenging behaviour.

\section{Biological factors}

\section{Genetic studies}

The determinants of some challenging behaviours will most likely closely reflect the genetics of the underlying disorder (e.g. vomiting in bulimia), and in others will reflect both genetic and epigenetic factors (e.g. violent behaviour in people with alcohol dependence or psychosis). Also, a number of genetic syndromes have been identified that are associated with varying degrees of specific maladaptive behaviour. Examples include Prader-Willi syndrome (compulsive over-eating) and Lesh-Nyhan syndrome (SIB). It has been proposed that study of these

Box 1 Definition of challenging behaviour

Challenging behaviour is a descriptive concept, which is largely socially constructed, and its meaning is subject to changes in social norms and service delivery patterns over time and across geographical areas

The term itself carries no diagnostic significance, and makes no inferences about the aetiology of the behaviour

It covers a heterogeneous group of behavioural phenomena across different groups of people; for example, oppositional behaviour in children, faecal smearing in those with a severe learning disability and deliberate self-harm in adult mental illness

Challenging behaviour may be unrelated to psychiatric disorder, but can also be a primary or secondary manifestation of it 
disorders may allow further understanding of the genetic contribution to particular behaviours. To this end, the concept of behavioural phenotypes has been introduced to describe the behavioural manifestations of a particular genetic make-up (genotype). However, there are a number of methodological problems in the study of behavioural phenotypes including lack of appropriate instruments, the subjective nature of behavioural observations and difficulty in identifying a behavioural standard. None the less, the study of some groups of people with genetically determined syndromes does allow a more fine-grained analysis of the genetic and epigenetic contributions to challenging behaviours in that group of people, and may also allow us to further understand the biological basis to psychiatric disorder in the general population. For example, people with velo-cardio-facial syndrome (VCFS) have a deletion at Q11 on chromosome 22 (the catechol-O-methyl transferase gene is in this region) and up to $30 \%$ of people with VCFS have psychosis.

\section{Neurochemical studies}

A number of endogenous substances have been investigated for their role in the development and maintenance of challenging behaviour. In particular, opioid peptides ( $\beta$-endorphins), sex hormones, dopamine and serotonin have been studied in relation to their role in mediating human behavioural processes such as aggression, arousal, selfinjury and appetite. Endogenous opioids have been implicated in the pathophysiology of SIB and a number of aetiological pathways have been hypothesised, for example, the intrinsically rewarding properties of endorphins released by SIB. Serotonin has been implicated in SIB, aggression, stereotypies, anxiety and behavioural disinhibition. Testosterone has been implicated in the mediation of aggressive and abnormal sexual behaviour. In particular, impulsive aggression in personality disorder correlates with tritiated paroxetine binding in the platelet. All the above-mentioned aetiological pathways have been utilised as the neurochemical basis of pharmacotherapeutic interventions.

\section{Brain structure and function}

The relationship between particular abnormalities in brain structure and function and well-defined challenging behaviours is poorly researched (for an overview, see Robertson \& Murphy, 1999). Thus, although there are many studies on neurobiological differences between people with psychiatric disorder and controls, there are relatively few which have related the frequency and severity of individual challenging behaviours to particular biological variables. None the less, some progress has recently been made, for example, in understanding the neurobiological correlates of violent behaviour. Lesion studies have implicated a number of brain areas in the regulation of aggression, including the amygdalahippocampal complex and prefrontal cortex (Mirsky \& Siegel, 1994). Qualitative computerised axial tomography (CT) and quantitative positron emission tomography (PET) studies have reported anatomical abnormalities, and reduced glucose metabolism, in prefrontal and temporal regions. However, until recently, nobody had related neurobiological variables to frequency of violence, or used quantitative in vivo techniques to investigate neuronal integrity and brain anatomy of people who are repetitively violent. Thus, we used proton magnetic resonance spectroscopy (HMRS) to study the neuronal integrity of the prefrontal lobe and amygdala-hippocampal complex in repetitively violent adults and nonviolent matched controls. We found that repetitively violent people had reduced neuronal density and abnormal phosphate metabolism in the prefrontal lobe and amygdala-hippocampal complex, and the degree of reduced neuronal density was related to frequency of violence (Critchley et al, 2000).

\section{Psychosocial factors}

A wide range of theoretical frameworks present conceptualisations of challenging behaviour, including psychoanalytic and sociological theories. However, clinical approaches derived from learning theory, which have been subject to the rigours of empirical validation, will be the main focus of this paper.

\section{The functionalist approach}

The majority of research into psychological factors underpinning the aetiology of challenging behaviour has taken a functional perspective, with its origins in learning theory. In this approach, the emphasis is on the purpose the behaviour serves for the individual, rather than the form of the behaviour per se. Alternative hypotheses relating to the functions of the target behaviour are developed (functional assessment) and can be evaluated systematically (functional analysis). In addition to accessing or avoiding either external or internal events, challenging behaviour may also serve as a form of communication. A single challenging behaviour can also be multi-functional (for a review see Sturmey, 1996) and there is an emphasis on internal events and emotion in the modern functional analysis.

Experimental research on challenging behaviours has, again, historically focused on people with learning disability, seeking to evaluate assessment and treatment procedures. For example, a large number of studies have investigated SIB, concluding 
that it is often a learned behaviour, acquired through an individual's history of interaction with his or her social and/or physical environment. Studies which have applied the methodology of functional analysis to large numbers of cases conclude it to be highly effective in identifying the environmental determinants of SIB on an individual basis (Iawata et al, 1994). Moreover, treatments are only effective when they match the functions of the target behaviour appropriately.

More recently, the methodology of functional analysis has been applied in populations without learning disability, for example, in conditions as diverse as anorexia nervosa, delusional speech and hallucinatory behaviour, problem-drinking and personality disorder.

\section{Assessment and treatment of challenging behaviour}

\section{Service delivery issues}

Challenging behaviours do not always come to the attention of health care professionals. A large proportion of people who exhibit challenging behaviour are dealt with by the penal system (e.g. prison rehabilitation or probation service), the educational system (e.g. educational psychologists or special schooling), or social services and the voluntary sector (e.g. supported housing, day and respite care provision). Specifically in the population with learning disability, challenging behaviour can be a significant obstacle to resettlement in the community and a frequent cause of requests for admission or readmission to hospital (Mansell, 1994). Currently, the National Health Service spends over $£ 600$ million on services for people with learning disability. In addition, local authorities spend approximately $£ 400$ million (Audit Commission, 1992). It is not known what proportion of this expenditure is spent on challenging behaviour services, but given the high prevalence of challenging behaviour in learning disability, with services needing to be highly resourced, it is likely to be a significant amount. When people with challenging behaviour, with or without learning disability, do receive health care, it is usually within the mental health care system - but the particular service model varies in location and treatment methods employed. Thus, services may be delivered by general adult psychiatric services (including community mental health teams, intensive-care units and challenging-behaviour units), neuropsychiatric services (including brain injury and epileptology services), learning disabilities teams, forensic psychiatric services and child and adolescent teams.

Both assessment and treatment constitute integral components of the management of patients with challenging behaviour, and the two processes may not be easily distinguishable - as continuous reassessment often merges with treatment procedure. Thus, the setting of the patient's management needs to be carefully considered. Out-patient treatment is the obvious first option, provided that safety issues are taken into account. It has the advantage of treating persons in their natural environment, thereby limiting the problems in generalisation of treatment response from in-patient to community settings. None the less, hospitalisation may be indicated because of the frequency and severity of the challenging behaviour, and specialist in-patient units offer an effective treatment option for certain groups of people (Xenitidis et al, 1999). The use of a legal framework under the Mental Health Act (MHA) may be necessary under certain circumstances, when the patient is thought to be suffering from one of the four categories of mental disorder (mental illness, psychopathic disorder, mental impairment and severe mental impairment). The importance that the legislation attaches to challenging behaviour is indicated by the fact that the three categories defined in Section 1 of the MHA (mental illness is not defined) require a behavioural criterion ("seriously irresponsible or abnormally aggressive behaviour") to be satisfied in order for the person to be thought of as suffering from one of these disorders. For mental impairment and severe mental impairment, the behaviour needs to "be associated with" the condition, whereas for psychopathic disorder the behaviour must be "the result of" the condition.

The general principles for the management of challenging behaviour are shown in Box 2 .

\section{General principles}

Whatever the setting and the legal framework, the management of challenging behaviour is typically resource-intensive. Often, a single agency may take the lead, but collaboration between a number of agencies will be necessary. Within each agency, a number of disciplines will need to be involved in both the assessment and the treatment phase. The collection of background information from a number of sources is essential if an accurate formulation of the development of the challenging behaviour is to be made, and the risk associated with it minimised. Detailed review of patients' medical, educational and social records is time-consuming but necessary for clarifying the degree of success of previous formulations and interventions. Medical investigations 
Box 2 Service issues in the management of challenging behaviour

Management needs to be tailored to the individual person, taking into account the particular behaviour and the setting in which it occurs

Multi-agency, multi-disciplinary involvement is necessary, and it is essential to gather detailed information about the nature and outcome of previous interventions

Different treatment modalities, i.e. pharmacotherapy, psychological and social interventions, alone or in combination, may be required. Only one treatment should be introduced at a time

The safety of the person displaying the challenging behaviour and of others must be considered carefully. A detailed risk assessment should be conducted, and the degree of urgency of response decided

Treatment in a safe and secure environment, if necessary within the framework of the Mental Health Act in a specialised unit, may need to be considered

will be required, as appropriate, into the presence of any comorbid medical condition or mental disorder. If a causative association is suspected, the direction of causality should be determined.

Information should be gathered from a variety of sources, including an individual's self-report, interview with family and carers, and direct observation of behaviour. Management should be guided by the principles of sequential single hypothesis testing. That is, the challenging behaviour should be quantified at baseline, and the effectiveness of each intervention assessed by accurately, and reliably, measuring pre- and post-treatment levels (Box 3). Only one treatment should be introduced at a time, and response to that treatment should be measured before it is replaced or augmented by others. In this way, the particular benefits of specific treatments can be evaluated, allowing later refinement of focused service delivery.

The use of standardised assessment tools facilitates the reliable and accurate measurement of the target behaviour. Several scales exist which provide comprehensive assessment of domains of challenging behaviour, for example, the Adaptive Behaviour Scale (Nihira et al, 1974). However, these were primarily developed for use with people with learning disability and do not generalise well to the general adult population. Although each clinical speciality may possess its own tailored measures to describe and categorise problems presented in that clinical area (e.g. Body Mass Index in eating disorders), people may also have a number of generic problems which require broader assessment tools (e.g. selfinjury or depression). For example, the Overt Aggression Scale (OAS) for the Objective Rating of Verbal and Physical Aggression (Yudofsky, 1986) allows verbal and physical aggression to others, self and property to be evaluated in a clinically applicable format. As noted above, the aim of this part of the assessment is to measure objectively the frequency and severity of the challenging behaviour and any putative determining factors.

Close attention must be paid to the reliability and validity of measures that are used, and the assessment should be multi-modal, rather than relying on a single source of information. Direct naturalistic observations of the behaviour, and related events, can be systematically recorded on A-B-C charts. Here, the antecedents (A), behaviour (B) and its consequences $(C)$ are described and the contingent relationships analysed. Behaviour can also be recorded using a range of idiosyncratic observational schemes. The most commonly employed are sampling schemes, where behaviour is observed and recorded at predefined temporal intervals. Intervals can be scored according to a number of rules. Observations of behaviour via such 'samples' are represented as directly proportional to 'real time'. Alternatively, behaviour can be observed via analogue conditions, where environmental events are systematically manipulated, and the effect on behaviour observed, to determine which reinforcers are operative in the natural environment.

Whatever the methods employed, information gathered should aim to:

(a) establish the properties (frequency, duration, etc.) of the behaviour at 'baseline' point in time;

Box 3 Steps towards systematic assessment and treatment of challenging behaviour

Identification of target behaviour(s)

Quantitative measurement of target behaviour Generation of hypotheses (medical, psychological and social) about the genesis and maintenance of the behaviour

Delivery of therapeutic intervention designed to test the hypotheses developed in line with a sequential, single hypothesis-testing model

Evaluation of effectiveness of the intervention Generation and testing of alternative hypotheses 
(b) develop and evaluate alternative hypotheses relating to the functions of the behaviour;

(c) predict the times and situations when the behaviour will and will not be performed across the full range of typical daily routines;

(d) define the function that the behaviour serves for the individual.

\section{Specific therapeutic interventions}

Pharmacotherapy

The treatment of an underlying mental disorder, epilepsy or other physical condition should be the target of any specific medication in the first instance. The general principles of pharmacotherapy should follow the principles highlighted in the section on Aetiology.

\section{Self-injury}

The opioid antagonists naloxone and naltrexone have been used for the reduction of SIB in learning disability patients. It is thought that this reduction is mediated by a selective blockade of endorphin receptors leading to removal of the biologically based reinforcing properties of self-injury. There have also been reports on the effective use of serotonergic antidepressants for the treatment of SIB.

\section{Sexually inappropriate behaviour}

Antilibidinal drugs such as cyproterone acetate have been used with recidivist sex offenders and other patients repeatedly exhibiting unacceptable or dangerous sexual behaviour. The mechanism of action of antilibidinal drugs is thought to be through a reduction of circulating androgens in the blood stream. As testosterone has been associated with aggressive as well as hypersexual behaviour, these drugs may act on both components of complex aggressive sexual behaviour, reducing its manifestation. Although all neuroleptic drugs have an antilibidinal effect that is usually regarded as a sideeffect of their use as antipsychotics, benperidol is thought to have a more marked antilibidinal effect, and sexually inappropriate behaviour is included in its formulary indications.

\section{Aggression}

Although some drugs have been marketed as having a specific anti-aggressive effect (lithium and chlorpromazine), it is more likely that any reduction of aggression is either secondary to a reduction of a primary psychopathology, or results from a nonspecific sedative effect (Box 4). Neuroleptics, benzodiazepines (caution is required because of
Box 4 Controversial issues in the management of challenging behaviour

Challenging behaviour is a socially constructed concept, and as such represents an entity of questionable validity

There exists no consensus about the borders between health and social care and what constitutes a health or social issue in relation to challenging behaviour

Interventions for challenging behaviour (including physical restraint, seclusion/ exclusion and programmes based on the principles of reinforcement) have the potential to be misused as punishment or a method of social control

The use of drug treatment for challenging behaviour with no clearly understood neurochemical basis, and with no evidence of underlying mental illness, may be both ethically and scientifically dubious

Use of the Mental Health Act for challenging behaviour may be open to abuse

Controversy about the most appropriate clinical speciality may result in patients 'falling between two stools'

possibility of paradoxical excitement), mood stabilisers and antidepressants have all been used for the treatment of aggressive behaviour.

\section{Psychological treatments}

From a learning theory perspective, Goldiamond (1974) outlines two contrasting approaches to the assessment and treatment of problematic behaviours. The pathological approach views challenging behaviour as a problem that has to be suppressed or removed. Although many studies report effective suppression of targeted challenging behaviour (e.g. by extinction), interventions based on a pathological approach can be described as 'prosthetic', and there are well-established problems with generalisation across settings, long-term maintenance and symptom substitution. The constructional approach views challenging behaviour as a successful means of serving a function. It can be seen in an individual whose resources are compromised as a legitimate and logical path to a desired natural consequence, albeit distressing to the person or others. Interventions based on a constructional approach focus on establishing new, less distressing behaviours, which 
will serve the same function, leading to the same natural consequence.

\section{Behaviour modification}

Treatment within a behaviour modification framework is based on the systematic analysis and application of reinforcement. Reinforcement is the process by which new responses are acquired and existing ones are strengthened. It refers to the procedure of providing consequences for the behaviour that increase or maintain the frequency of that behaviour. A reinforcer is defined by its results. Reinforcement programmes can manipulate the schedule, ratio and nature of reinforcers. Restructuring the environment to remove significant contingent events may also be viewed as necessary; for example, social relationships associated with patterns of addictive behaviour may be avoided until the individual feels confident about renewing acquaintances without reverting to former patterns of behaviour.

Assessment of challenging behaviour using functional analysis can help to identify for the individual alternative behaviours that will produce desirable consequences similar to those of the challenging behaviour. The person is then encouraged to substitute these alternative behaviours in settings that would usually elicit the target behaviour. This is called solution analysis and is a treatment approach of established therapeutic value. For example, it has been demonstrated that functional and subsequent solutional analyses of parasuicidal acts result in fewer incidents of parasuicidal behaviour and fewer in-patient days than standard therapies (Linehan et al, 1993). The skills inherent in producing the alternative behaviour may need to be learned/shaped, as they may not be present in the individual's repertoire.

\section{Cognitive-behavioural therapy}

The cognitive model and its practical application in clinical psychology have allowed psychological interventions to become more targeted in their use of internally generated material as a focus for treatment. Cognitive-behavioural therapy (CBT) encompasses a wide range of interventions, offered to clients within the framework of 'collaborative empiricism'. As well as behavioural strategies for managing symptoms, thoughts, beliefs and feelings are perused and examined for relevance and validity. Traditional or 'elegant' cognitive therapy was developed to work either in combination with pharmacotherapy or alone to ameliorate the symptoms of depression in adults. The success of CBT as an effective intervention has encouraged clinicians and researchers to investigate and apply its techniques in a wide range of clinical problems; these include depression (for a review, see Watkins \& Williams, 1998), panic (Clark et al, 1994), psychotic symptoms (Garety et al, 1994) and personality disorder (Nelson-Gray \& Farmer, 1999). More recently, the efficacy of CBT as a treatment for adults with mild learning disability has been established (Lindsay et al, 1993), although, to date, sample sizes have been small.

\section{Psychodynamic and systemic therapies}

Individual, family and group psychotherapeutic approaches based on systemic or psychodynamic theories have been used in the treatment of challenging behaviour, either alone or as an adjunct to other therapeutic modalities. Data on their effectiveness and efficacy are limited, especially in the learning disability field. Although a number of case reports and review articles have been published in recent years, especially in the area of forensic psychotherapy and psychological treatments of people with personality disorders, further research on both the process and the outcome of psychotherapeutic approaches is needed.

\section{References}

Audit Commission (1992) Community Care: Managing the Cascade of Care. London: HMSO.

Borthwick-Duffy, S. A. (1994) Prevalence of destructive behaviors. In Destructive Behavior in Developmental Disabilities: Diagnosis and Treatment (eds T. Thompson \& D. B. Gray), pp. 3-23. Thousand Oaks, CA: Sage.

Clark, D. M., Salkovskis, P. M., Hackman, A., et al (1994) A comparison of cognitive therapy, applied relaxation and imipramine in the treatment of panic disorder. British Journal of Psychiatry, 164, 759-769.

Critchley, H., Simmons, A., Daly, E., et al (2000) Prefrontal and medial temporal correlates of repetitive violence to self and others. Biological Psychiatry, 47, 928-934.

Emerson, E. (1995) Challenging Behaviour. Analysis and Intervention in People with Learning Difficulties. Cambridge: Cambridge University Press.

Garety, P. A., Kuipers, E., Fowler, D., et al (1994) Cognitivebehavioural therapy for drug-resistant psychosis. British Journal of Medical Psychology, 67, 259-271.

Goldiamond, I. (1974) Toward a constructional approach to social problems. Behaviourism, 1, 1-84.

Iawata, B. A., Pace, G. M., Dorsey, M. F., et al (1994) The functions of self-injurious behaviour: an experimental epidemiological analysis. Journal of Applied Behaviour Analysis, 27, 215-240.

Lindsay, W. R., Howells, L. \& Pitcaithly, D. (1993) Cognitive therapy for depression with individuals with intellectual disabilities. British Journal of Medical Psychology, 66, 135141

Linehan, M., Heard, H. \& Armstrong, H. (1993) Naturalistic follow-up of a behavioural treatment for chronically parasuicidal borderline patients. Archives of General Psychiatry, 50, 971-974.

Mansell, J. (1994) The challenge of providing high quality services. In Mental Health in Mental Retardation (ed. N. Bouras), pp. 328-340. Cambridge: Cambridge University Press. 
Menezes, P. R., Johnson, S., Thornicroft, G., et al (1996) Drug and alcohol problems among individuals with several mental illness in south London. British Journal of Psychiatry, 168, 612-619.

Mirsky, A. F. \& Siegel, A. (1994) The neurobiology of violence and aggression. In Understanding and Preventing Violence. Vol. II Biobehavioural Influences, pp. 59-172. Washington, DC: National Academic Press.

Nelson-Gray, R. O. \& Farmer, R. F. (1999) Behavioural assessment of personality disorders. Behaviour Research and Therapy, 37, 347-368.

Nihira, K., Foster, R., Shellhaas, M., et al (1974) AAMD Adaptive Behaviour Scale. Washington, DC: American Association of Mental Deficiency.

Qureshi, H. \& Alborz, A. (1992) Epidemiology of challenging behaviour. Mental Handicap Research, 5, 130-145.

Regier, D. A., Narrow, W. E., Rae, D. S., et al (1993) The de facto US mental and addictive disorders service system. Epidemiologic catchment area prospective 1-year prevalence rates of disorders and services. Archives of General Psychiatry, 50, 85-94.

Robertson, D. \& Murphy, D. G. M (1999) Brain imaging and behaviour. In Psychiatric and Behavioural Disorders in Developmental Disabilities and Mental Retardation (ed. N. Bouras). Cambridge: Cambridge University Press.

Steinart, T., Wiebe, C. \& Gebhardt, R. P. (1999) Aggressive behaviour against self and others among first-admission patients with schizophrenia. Psychiatric Services, 50, 85-90.

Sturmey, P. (1996) Functional Analysis in Clinical Psychology. Chichester: John Wiley \& Sons.

Watkins, E. \& Williams, R. (1998) The efficacy of cognitivebehavioural therapy. In The Management of Depression (ed. S. Checkley). Oxford: Blackwell.

Xenitidis, K. I., Henry, J., Russell, A. J., et al (1999) An inpatien treatment model for adults with mild intellectual disability and challenging behaviour. Journal of Intellectual Disability Research, 43, 128-134.

Yudofsky, S. C., Silver, J. M., Jackson, W., et al (1986) The Overt Aggression Scale for the objective rating of verbal and physical aggression. American Journal of Psychiatry, 143, 35-39.

\section{Multiple choice questions}

1. The term challenging behaviour:

a implies unknown aetiology

b is reserved for people with a learning disability

c cannot be used for people with mental illness

$\mathrm{d}$ is socially constructed

e does not apply to people with autism.

2. Treatment of challenging behaviour:

a should avoid pharmacotherapy

$\mathrm{b}$ is nearly always multi-disciplinary

c an in-patient setting should be avoided

d should avoid the simultaneous introduction of two treatment modalities

e depends on underlying aetiology.
3. Out-patient treatment of challenging behaviour:

a may be too risky to undertake

$\mathrm{b}$ is nearly always the preferred option

c should always be used with adolescents

d prohibits use of psychotherapeutic techniques

e is never feasible with offenders.

4. In the management of challenging behaviour, the Mental Health Act:

a should be used only if there is an underlying illness

$\mathrm{b}$ is not applicable to adolescents

c is applicable in severe learning disability

$\mathrm{d}$ is only applicable to offenders

$\mathrm{e}$ is almost always ethically dubious.

5. The constructional approach to challenging behaviour implies:

a providing negative consequences for the behaviour

b viewing the behaviour as successfully serving a function

c highlighting further education

d aiming to replace the challenging behaviour with behaviour serving the same function

e defining the pattern of reinforcement.

$\begin{array}{lllllllllll}\text { MCQ answers } & & & & & & & \\ \text { 1 } & & 2 & & 3 & & 4 & & & 5 & \\ \text { a } & \text { F } & \text { a } & \text { F } & \text { a } & \text { T } & \text { a } & \text { F } & \text { a } & \text { F } \\ \text { b } & \text { F } & \text { b } & \text { T } & \text { b } & \text { T } & \text { b } & \text { F } & & \text { b } & \text { T } \\ \text { c } & \text { F } & \text { c } & \text { F } & \text { c } & \text { F } & \text { c } & \text { T } & \text { c } & \text { F } \\ \text { d } & \text { T } & \text { d } & \text { T } & \text { d } & \text { F } & \text { d } & \text { F } & \text { d } & \text { T } \\ \text { e } & \text { F } & \text { e } & \text { T } & \text { e } & \text { F } & \text { e } & \text { F } & \text { e } & \text { F }\end{array}$

